\title{
Need to improve awareness and management of hepatitis $B$ reactivation in patients receiving immunosuppressive therapy
}

\author{
Man-Fung Yuen ${ }^{1,2}$
}

Received: 25 August 2015/Accepted: 7 December 2015/Published online: 6 January 2016

(C) Asian Pacific Association for the Study of the Liver 2016

\begin{abstract}
Hepatitis B reactivation in patients receiving immunosuppressive therapy is not an uncommon event because of upsurge in the development of more potent and new immunosuppressive therapy (IST) including monoclonal antibodies leading to profound B cell depletion. However, this condition is still not being commonly made known to practising clinicians. More importantly, serious adverse outcomes including liver decompensation and death have been reported from time to time. This is likely related to the insufficient efforts to disseminate the knowledge or inattentive attitude from clinicians towards the need for hepatitis B virus screening before starting IST. It is of crucial importance, as these adverse outcomes are largely preventable by prophylactic or early treatment by antiviral agents. The risk of hepatitis $\mathrm{B}$ reactivation depends on the type and duration of the IST, patient characteristics and disease conditions. While we are eagerly waiting for a more cohesive consensual management recommendations from the different specialties who are involved in managing these patients, consolidated data are readily available for clinicians to define whether their patients would have a low or a high risk of hepatitis B reactivation. A management strategy without ambiguity should be formulated.
\end{abstract}

Man-Fung Yuen

mfyuen@hkucc.hku.hk

1 Department of Medicine, Queen Mary Hospital, The University of Hong Kong, Pokfulam Road, Hong Kong, China

2 State Key Laboratory for Liver Research, The University of Hong Kong, Hong Kong, China

\section{Introduction}

Hepatitis B virus (HBV) reactivation in chronic hepatitis B patients undergoing chemotherapy/immunosuppressive therapy (IST) was first described in the literature in 1975 by Wands et al. [1]. It was found that $\mathrm{HBV}$ reactivation could occur in both hepatitis B surface antigen (HBsAg)positive and -negative patients. The latter is now usually referred as patients with resolved infection or patients with occult hepatitis B infection (OBI). The occurrence of HBV reactivation is related to several possible mechanisms. HBV replication increases as a result of the stimulation of the glucocorticoid responsive element of the viral genome through the use of a steroid as one of the components of the IST/chemotherapy. IST may also repress lymphocyte functions and inhibit the production of effector cytokines, e.g., tumor necrosis factor alpha and interferon gamma [2]. Finally, with the immune rebound after withdrawal of the IST, immune-mediated hepatic damage occurs and leads to hepatic inflammation. HBV reactivation may end up with an unremarkable course, but can also cause irreversible liver damage leading to hepatic decompensation and even mortality. The outcome of $\mathrm{HBV}$ reactivation depends on the severity of the flares and also the baseline functional status of the liver, e.g., the presence of advanced fibrosis and cirrhosis.

\section{Awareness and perception}

According to a study in 2012 surveying practising oncologists in the United States, $20 \%$ of them would not perform any type of screening for HBV before the start of IST [3]. The negligence of this important issue is further underlined by the finding that less than $40 \%$ of them 
would perform screening for HBV even in patients who were known to have high-risk factors for HBV infection or a history of hepatitis.

This unsatisfactory phenomenon seems to be improving according to an Italian questionnaire study reported in 2014 [4]. There was a higher level of awareness of the issue of HBV reactivation in specialists managing hematological malignancies. Over $91 \%$ of them were aware of the problem of HBV reactivation as a consequence of IST. More than $93 \%$ of them would perform screening for HBV. It is, however, not known whether the conclusion obtained through questionnaire survey reflects the actual practising situation in the real world setting. A recent study done in United States, published in the issue of this journal, showed that of the 17 patients with HBV reactivation secondary to IST admitted/transferred to a tertiary center over the last 15 years, only 3 patients were screened for HBV prior to IST [5]. Because of the lack of awareness of the problem, 15 patients were only prescribed antiviral agents 1-60 days after admission. Consequently, there were 3 deaths, including 1 of the 2 patients who had received liver transplantation. The study also examined the temporal figure of hospital admission over 15 years. It was found that there was no trend of decline of hospital admission due to HBV reactivation. This indirectly suggested that awareness of prevention of HBV reactivation by the most effective means of HBV screening prior to IST has not increased among clinicians over the years.

An even more worrisome situation is that only $17 \%$ of patients received $\mathrm{HBV}$ testing before initiation of chemotherapy in a study from China [6], a region with high prevalence of HBV disease. It is of urgent and paramount importance to increase clinicians' awareness and perception of the potential life-threatening outcome of $\mathrm{HBV}$ reactivation, an adverse outcome which is totally avoidable, in patients receiving IST.

\section{Current knowledge of $\mathrm{HBV}$ reactivation}

\section{Rate of $\mathrm{HBV}$ reactivation}

The chance of HBV reactivation obviously depends on (1) types and intensity of IST, (2) the duration of IST, and (3) the patient population. There are very few prospective studies to examine the chance of $\mathrm{HBV}$ reactivation in OBI patients. Most of the studies examining the rate of HBV reactivation were performed in $\mathrm{HBsAg}$-positive populations. It was found that, compared to standard cytotoxic chemotherapy without corticosteroid, the addition of corticosteroid increased the incidence from 38 to $73 \%$ [7]. According to some studies on biologics, anti-TNF was associated with an approximately $15-35 \%$ chance of HBV reactivation in $\mathrm{HBsAg}$-positive patients [8]. For OBI subjects, the incidence was found to be $3 \%$. For the currently commonly used anti-CD 20, rituximab, the HBV reactivation incidence increased by fivefold [9]. According to two recent studies, the incidence of HBV reactivation in HBsAg-positive patients receiving rituximab was around $40-59 \%$ [10, 11].

\section{Factors affecting the rate of $\mathrm{HBV}$ reactivation}

\section{Patient gender and underlying malignancy}

It has been shown that male patients had a higher chance of HBV reactivation compared to female patients [12]. Patients with lymphoma had a higher risk of HBV reactivation compared to other types of malignancy. However, the intensity of immunosuppression exerted by different regimens of IST used in different types of malignancy may be the confounding factor.

\section{Categories of immunosuppressive therapy}

There are at least 5 groups of diseases in which IST is commonly used as standard therapy. These include malignancy (solid tumor), hematological conditions including malignancy and hematopoietic stem cell transplantation (HSCT), gastrointestinal diseases, rheumatological/autoimmune diseases and dermatological diseases. IST is also used in patients receiving organ transplantation to prevent rejection.

In addition to steroids, among the common chemotherapy agents, the anthracycline group is associated with a higher chance of HBV reactivation. There are now newer groups of agents which are associated with HBV reactivation. These include (1) biologics, in particular, anti-tumor necrosis factor (anti-TNF), e.g., infliximab, adalimumab and etanercept, and (2) monoclonal antibodies against B cell surface antigens, anti-CD 20, e.g., rituximab and ofatumumab. Apart from the specific agents used in the IST, the duration of IST is also an important factor for HBV reactivation. Prolonged immunosuppressive therapy, e.g. in HSCT, is associated with a more long-standing risk of $\mathrm{HBV}$ reactivation. According to a study enrolling HBsAg-negative and anti-HBc-positive patients, the cumulative rates of $\mathrm{HBsAg}$ seroreversion were 13 and $22 \%$ at 1 and 5 years of follow-up, respectively [13].

\section{HBV serology}

\section{HBsAg-positive patients}

Because of the considerable high rate of HBV reactivation mentioned above, it is universally recommended that HBsAg-positive patients undergoing IST should be 
Table 1 Incidence of HBV reactivation in patients with different HBV serology and receiving different immunosuppressive therapy

\begin{tabular}{lll}
\hline & \multicolumn{2}{l}{ Incidence of HBV reactivation } \\
\cline { 2 - 3 } & $\begin{array}{ll}\text { HBsAg-positive subjects } \\
(\%)\end{array}$ & $\begin{array}{l}\text { HBsAg-negative, anti-HBc-positive } \\
\text { subjects }(\%)\end{array}$ \\
\hline Systemic chemotherapy & $24^{\mathrm{a}}-48^{\mathrm{b}}$ & $<3^{\mathrm{d}}$ \\
Rituximab/steroid containing regimens & $59^{\mathrm{c}}$ & $8^{\mathrm{e}}-42^{\mathrm{f}}$ \\
HSCT/organ transplantation & $>50^{\mathrm{d}}$ & $37^{\mathrm{g}}$ \\
\hline
\end{tabular}

HSCT haematopoietic stem cell transplantation

${ }^{a}$ See Ref. [23]

b See Ref. [24]

c See Ref. [11]

d See Ref. [25]

e See Ref. [16]

${ }^{f}$ See Ref. [14]

g See Ref. [17] prophylactically treated with anti-viral agents. However, the duration of coverage with the antiviral therapy for patients for whom the baseline HBV DNA is lower than $2000 \mathrm{IU} / \mathrm{mL}$ and/or without serious liver disease including advanced liver fibrosis or cirrhosis, is still controversial. In general, antiviral therapy should be continued for at least 6 months after conventional chemotherapy and at least 12 months after rituximab-containing IST, although HBV reactivation has been seen even after 12 months of cessation of rituximab $[14,15]$. Because of the prolonged immunosuppression in HSCT, a longer duration of antiviral therapy is usually required.

\section{HBsAg-negative and anti-HBc-positive patients}

According to recent studies $[14,16]$, the HBV reactivation rate in anti-HBc positive patients receiving rituximabcontaining IST ranges from 8.3 to $42 \%$. A preliminary study of HSCT anti-HBc positive patients showed that the HBV reactivation was $37 \%$ [17]. In studies examining anti-TNF, the HBV reactivation rate was less than $5 \%$ [8, 18]. Across all studies, the association between high titers of anti-HB level and a low rate of HBV reactivation were consistently shown [14, 19-21]. However, recommendations using anti-HB level to define high or low risk of HBV reactivation are not yet firmly established.

A consensual approach for HBsAg-negative, anti-HBcpositive patients has not been reached between hepatologists and oncologists. Most clinicians would adopt a regular 4-12 weekly "HBV DNA monitoring" and initiate treatment with antiviral therapy when there is detectable HBV DNA. This has been proven to be successful, as there are no hepatitis events when treatment is given at this stage. Other clinicians would adopt a prophylactic approach even when HBV DNA is not detectable, especially for patients receiving high-risk IST regimens. There exist no good data on the outcome of patients who receive no treatment when the HBV DNA levels are low, e.g., $<2000 \mathrm{IU} / \mathrm{mL}$ at the time of HBV reactivation. The clinical significance of transient low HBV DNA during/after IST treatment is not known. Some patients have very low transient detectable HBV DNA which would become undetectable in subsequent follow-up without administration of antiviral treatment.

\section{HBsAg-negative and anti-HBc-negative patients}

Patients who are negative for both $\mathrm{HBsAg}$ and anti-HBc are "safe" patients in term of the chance of HBV reactivation, although there exists an entity of sero-negative OBI. The chance of $\mathrm{HBV}$ reactivation is quite low. A more cautious approach is to monitor the HBV DNA only in patients receiving high-risk IST, e.g., in patients receiving rituximab and in patients with HSCT. However, the interval of monitoring is yet to be decided.

The incidence of HBV reactivation in patients with different HBV serology and receiving different IST regimens is listed in Table 1.

\section{Choice of antiviral therapy}

As the first approved oral antiviral agent, lamivudine has been used as a prophylactic agent to reduce the rate of HBV reactivation in patients undergoing IST. It has been shown that the risk of HBV reactivation could be reduced by $79 \%$ when lamivudine was used in these patients [2]. Because of its disadvantage of a high rate of viral mutation and the availability of more potent antiviral agents with very low rates of viral resistance development, entecavir and tenofovir are now replacing lamivudine in many centers. According to a study in China, the use of entecavir 
caused a further $23 \%$ reduction of HBV reactivation and a $13 \%$ reduction of $\mathrm{HBV}$-related hepatitis when compared with lamivudine [22]. It was also associated with less disruption of chemotherapy. The more favorable outcome of using entecavir compared to lamivudine is also supported by another Korean study [10].

\section{Conclusions}

HBV reactivation has become an important problem in overt or occult HBV patients receiving IST, especially in the era of more potent anti-cancer and B cell depletion therapeutic agents. The risk has been defined with more solid evidence from prospective studies. Efforts should be made in disseminating this knowledge to practising hepatologists and oncologists. The emphasis should be on preIST screening for $\mathrm{HBsAg}$ and anti-HBc, and on treating or monitoring HBV DNA levels during and after the IST. By implementation of this recommendation, the occurrence of hepatitis flares, hepatic decompensation and liver mortality can be largely prevented.

\section{References}

1. Wands JR, Chura CM, Roll FJ, et al. Serial studies of hepatitisassociated antigen and antibody in patients receiving antitumor chemotherapy for myeloproliferative and lymphoproliferative disorders. Gastroenterology. 1975;68:105-12

2. Loomba R, Rowley A, Wesley R, et al. Systematic review: the effect of preventive lamivudine on hepatitis $\mathrm{B}$ reactivation during chemotherapy. Ann Intern Med. 2008;148:519-28

3. Kawsar HI, Shahnewaz J, Gopalakrishna KV, et al. Hepatitis B reactivation in cancer patients: role of prechemotherapy screening and antiviral prophylaxis. Clin Adv Hematol Oncol. 2012; 10:370-8

4. Marignani M, Marzano A, Begini P, et al. Perception of hepatitis $B$ virus infection reactivation-related issues among specialists managing hematologic malignancies: result of an Italian survey. Leuk Lymphoma. 2014;55:2564-71

5. Patel A, Yapali S, Lok ASF. Admissions for hepatitis B reactivation in patients receiving immunosuppressive therapy remain unchanged from 1999 to 2014. Hepatol Int 2016

6. Wang Y, Luo XM, Yang D, et al. Testing for hepatitis B infection in prospective chemotherapy patients: a retrospective study. World J Gastroenterol. 2013;19:923-30

7. Cheng AL, Hsiung CA, Su IJ, et al. Steroid-free chemotherapy decreases risk of hepatitis $\mathrm{B}$ virus (HBV) reactivation in $\mathrm{HBV}-$ carriers with lymphoma. Hepatology. 2003;37:1320-8

8. Pérez-Alvarez R, Díaz-Lagares C, García-Hernández F, et al. Hepatitis $\mathrm{B}$ virus (HBV) reactivation in patients receiving tumor necrosis factor (TNF)-targeted therapy: analysis of 257 cases. Medicine (Baltimore). 2011;90:359-71

9. Evens AM, Jovanovic BD, Su YC, et al. Rituximab-associated hepatitis $\mathrm{B}$ virus (HBV) reactivation in lymphoproliferative diseases: meta-analysis and examination of FDA safety reports. Ann Oncol. 2011;22:1170-80

10. Shih CA, Chen WC, Yu HC, et al. Risk of severe acute exacerbation of chronic HBV infection cancer patients who underwent chemotherapy and did not receive anti-viral prophylaxis. PLoS ONE. 2015; 18:e0132426

11. Kim SJ, Hsu C, Song YQ, et al. Hepatitis B virus reactivation in B-cell lymphoma patients treated with rituximab: analysis from the Asia Lymphoma Study Group. Eur J Cancer. 2013;49: 3486-96

12. Yeo W, Chan PK, Zhong S, Ho WM, et al. Frequency of hepatitis $\mathrm{B}$ virus reactivation in cancer patients undergoing cytotoxic chemotherapy: a prospective study of 626 patients with identification of risk factors. J Med Virol. 2000;62:299-307

13. Viganò M, Vener C, Lampertico P, et al. Risk of hepatitis B surface antigen seroreversion after allogeneic hematopoietic SCT. Bone Marrow Transplant. 2011;46:125-31

14. Seto WK, Chan TS, Hwang YY, et al. Hepatitis B reactivation in patients with previous hepatitis $\mathrm{B}$ virus exposure undergoing rituximab-containing chemotherapy for lymphoma: a prospective study. J Clin Oncol. 2014;32:3736-43

15. Yeo W, Chan TC, Leung NW, et al. Hepatitis B virus reactivation in lymphoma patients with prior resolved hepatitis B undergoing anticancer therapy with or without rituximab. J Clin Oncol. 2009;27:605-11

16. Kusumoto S, Tanaka Y, Suzuki R, et al. Monitoring of hepatitis B virus (HBV) DNA and risk of $\mathrm{HBV}$ reactivation in B-Cell Lymphoma: a prospective observational study. Clin Infect Dis. 2015;61:719-29

17. Seto WK, Chan SY, Hwang YY, et al. A prospective study of hepatitis $\mathrm{B}$ reactivation in patients with prior HBV exposure undergoing hematopoietic stem cell transplantation: reactivation association with graft-versus-host disease. J Hepatol. 2015;62(Suppl):S187

18. Lee $\mathrm{YH}, \mathrm{Bae} \mathrm{SC}$, Song GG. Hepatitis B virus (HBV) reactivation in rheumatic patients with hepatitis core antigen (HBV occult carriers) undergoing anti-tumor necrosis factor therapy. Clin Exp Rheumatol. 2013;31:118-21

19. Hammond SP, Borchelt AM, Ukomadu C, et al. Hepatitis B virus reactivation following allogeneic hematopoietic stem cell transplantation. Biol Blood Marrow Transplant. 2009;15:1049-59

20. Kanaan N, Kabamba B, Maréchal C, et al. Significant rate of hepatitis $\mathrm{B}$ reactivation following kidney transplantation in patients with resolved infection. J Clin Virol. 2012;55:233-8

21. Onozawa M, Hashino S, Izumiyama K, et al. Progressive disappearance of anti-hepatitis B surface antigen antibody and reverse seroconversion after allogeneic hematopoietic stem cell transplantation in patients with previous hepatitis B virus infection. Transplantation. 2005;79:616-9

22. Huang H, Li X, Zhu J, et al. Entecavir vs lamivudine for prevention of hepatitis B virus reactivation among patients with untreated diffuse large B-cell lymphoma receiving R-CHOP chemotherapy: a randomized clinical trial. JAMA. 2014;312:2521-30

23. Yeo W, Chan PK, Ho WM, et al. Lamivudine for the prevention of hepatitis $\mathrm{B}$ virus reactivation in hepatitis $\mathrm{B}$ s-antigen seropositive cancer patients undergoing cytotoxic chemotherapy. J Clin Oncol. 2004;22:927-34

24. Lok AS, Liang RH, Chiu EK, et al. Reactivation of hepatitis B virus replication in patients receiving cytotoxic therapy. Report of a prospective study. Gastroenterology. 1991;100:182-8

25. Kusumoto S, Tanaka Y, Mizokami M, et al. Reactivation of hepatitis B virus following systemic chemotherapy for malignant lymphoma. Int J Hematol. 2009;90:13-23 